\title{
Contribution à la connaissance des cours d'eau du Liban : la zonation biologique du Nahr Qab Ilias
}

\author{
N.J. Alouf 1
}

Les parties temporaire et permanente d'un cours d'eau du Liban central sont étudiées. Les données climatiques, hydrogéologiques, hydrographiques et biotiques du Nahr Qab Ilias mettent en relief la présence de trois zones étagées en fonction de l'altitude. Les Plécoptères dominent à l'amont et les Ephéméroptères à l'aval.

\section{Studies on Lebanese streams : the biological zonation of the Nahr Qab Illas.}

Intermittent and permanent parts of a chalky stream are studied. Climatical, hydrogeological, hydrographic and biological data are given. The stream is divided in three sectors. The Plecoptera are the main group of the zoocenosis in the upper part. The Ephemeroptera dominate in the lower part.

Dans le cadre de la promotion de la recherche scientifique, le C.N.R.S. du Liban nous a chargés de l'étude des macro-invertébrés des eaux courantes. La réalisation de ce projet s'est heurtée, dès le début, à deux difficultés majeures ; d'une part la reconnais. sance des formes vivantes et d'autre part l'absence d'études synthétiques sur les cours d'eau libanais. Il fallait, pour surmonter ces deux obstacles, entreprendre un travail de longue haleine : la récolte d'un grand nombre d'individus à différents niveaux et endroits durant toutes les périodes de l'année. C'est ce que nous nous sommes proposés d'accomplir.

Le bassin hydrographique de la rivière de Qab Ilias s'est le mieux prêté à une telle étude. Riche en différentes sortes de milieux dulçaquicoles, il est assez facilement accessible et il est possible de le prospecter pendant toute l'année. Nous esperons établir, à partir des relevés climatiques, hydrogéologiques et hydrographiques et de la liste faunistique, un modèle de zonation et de classification des eaux courantes au Liban, du moins celles d'altitude.

1. Faculté des Sciences, section I, Université Libanaise, Hadath Beyrouth, Liban (projet $n^{\circ} 51008$ du C.N.R.S. du Liban).
Faute de connaissances approfondies des différent s groupes zoologiques, l'étude de la zonation biologique se fera en fonction de la distribution des Plécoptères et des Ephéméroptères. Les travaux d'Elliott (1967), d'Ulfstrand (1968) et de Lavandier et Dumas (1971) montrent que les Plécoptères dominent les Ephéméroptères dans des cours d'eau d'Angleterre, de Scandinavie et de France (Tableau IV). La classification biologique des cours d'eau (Verneaux et Tuffery, 1967) considère la présence des Plécoptères et des Ephéméroptères Ecdyonuridés comme indicateurs des eaux très propres, zones I et II, les autres Ephéméroptères caractérisant la zone III.

Le travail de Legier et Talin (1973) compare des ruisseaux permanents et temporaires de la Provence calcaire. La colonisation des cours d'eau temporaires par les rhéophiles est dépendante de la curée d'écoulement des ruisseaux et de sa concordance avec celle de la phase larvaire.

Nous proposons de comparer la structure biotique du Nahr Qab Ilias dans ses phases temporaires et permanentes et d'essayes de déduire, par comparaison avec les travaux cités plus haut, une zonation biologique répondant aux conditions écologiques qui prévalent au Liban. 


\section{1. - Climatologie et pluviométrie}

Le climat méditerranéen rencontré ici est caractérisé par des chutes de pluie durant sept mois de l'année, suivies d'une sécheresse quasi absolue de mai jusqu'en octobre. La hauteur des pluies atteint $1000 \mathrm{~mm}$ à $1000 \mathrm{~m}$ d'altitude et plus de $1500 \mathrm{~mm}$ au-delà de $1400 \mathrm{~m}$. Les précipitations sont sous forme de neige, en hiver, sur la plus grande partie du bassin. Il tombe en janvier, le mois le plus humide, $150 \mathrm{~mm}$ d'eau à $1000 \mathrm{~m}$ et $200 \mathrm{~mm}$ à $1500 \mathrm{~m}$, en moyenne. Le cours d'eau est alimenté par voie de surface pendant la saison humide et par voie souterraine pendant la saison sèche.

\section{2. - Hydrogéologie}

Le bassin hydrographique de la rivière de $\mathrm{Qab}$ Ilias (Nahr Qab Ilias) est formé et alimenté par le mont Kunayssah (Kneissé) au nord, qui est un karst cénomanien, et le mont Barouk au sud, qui est un karst jurassique. L'aptien-albien forme la partie médiane entre les deux massifs ; on y trouve du grès, du calcaire et des marnes. La grande faille de Yammounah, de direction N.S, limite le mont Liban. D'autres failles, de moindre importance, sont orientées E-W ; elles limitent le karst jurassique, du sud, et fractionnent l'aptien-albien du centre. Le cours d'eau emprunte la plus importante faille de cet ensemble (fig. 1).

La superficie du bassin versant est de $15 \mathrm{~km}^{2}$. Il cornmence à $2000 \mathrm{~m}$ d'altitude, au nord, et à $1500 \mathrm{~m}$, au sud.

Le confluent avec le Litani est à $860 \mathrm{~m}$. Beaucoup de petites sources prennent naissance dans les couches aptiennes-albiennęs; elles se reconnaissent, en été, grâce aux taches de verdure. Les bergers entretiennent, assez fréquemment, de petits bassins dont la superficie ne dépasse pas quelques mètres car. rés et qui sont assez riches en faune.

Les torrents de montagne sont nettement plus longs et ramifiés du côté du massif Kunayssah que celui du Barouk. La plupart ne coulent que pour 2 3 mois. Le cours principal est actif durant 4-5 mois en-deçà de $1300 \mathrm{~m}$ et devient permanent à partir de $1000 \mathrm{~m}$. La largeur du lit est de $2-3 \mathrm{~m}$ à $1170 \mathrm{~m}$ d'altitude. Le cours d'eau emprunte, à ce niveau, des gorges innaccessibles taillées dans le jurassique.
Une partie de l'eau s'engouffre pour réapparaître plus bas et une autre continue par voie de surface. Par con séquent, le ruisseau fonctionne 6.7 mois à $1100 \mathrm{~m}$. Les résurgences commencent à apparaître vers $1025 \mathrm{~m}$, elles deviennent de plus en plus nom. breuses au fur et à mesure que l'on descend, jusqu'à former le Nahr Qab Ilias.

Le massif cénomanien, au nord, alimente essentiellement le bassin, durant la saison humide, par des eaux de ruissellement provenant soit des pluies,

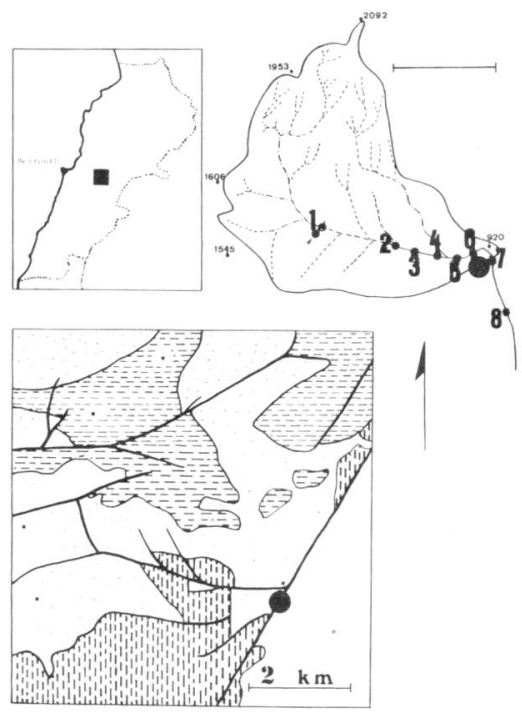

Fig. 1. En haut à gauche, carte du Liban. Le rectangle noir correspond à la région prospectée.

En haut a droite, bassin hydrographique de la rivière Qab Ilias. Les grands chiffres $(1$ a 8$)$ indiquent l'emplacement des stations prospectées; les petits chiffres, les cotes altitudinales en mètres : le cercle noir entre les stations 6 et 7 figure la ville de Qab Ilias. Le trait horizontal correspond a $2 \mathrm{~km}$.

En bas, carte géologique (même échelle). Le Jurassique est figuré par des hachures horizontales, le Cénomanien par des hachures verticales, l'AptienAlbien par des pointillés, les alluvions quatemaires par un espace blanc. Les traits épais correspondent aux failles. 
soit de la fonte des neiges. Le massif jurassique, au sud, l'alimente par des eaux de ruissellement mais. surtout, par des eaux souterraines karstiques. Le jurassique du Barouk est considéré comme un châ. teau d'eau ; le ruisseau ne devient permanent qu'à son niveau.

\section{3. - Données hydrographiques}

Le bassin hydrographique de la rivière Qab Ilias occupe une partie médiane sur le versant est de la chaine du Liban, depuis la ligne des crêtes jusqu'à son confluent avec le fleuve Litani (fig. 1). Nous l'avons divisé en trois secteurs:

1. - Secteur montagnard. Depuis la formation des premiers filets d'eau saisonniers jusqu'à la zone des sources pérennes vers $1020 \mathrm{~m}$.

2. - Secteur submontagnard. Entre la zone des sources pérennes et la ville de Qab Ilias à $920 \mathrm{~m}$.

3. - Secteur inframontagnard. La rivière, depuis Qab Ilias jusqu'au confluent avẹc le Litani à $860 \mathrm{~m}$.

Le débit, au niveau de la ville, est assez régulier, avec un maximum hiverno-printanier et un minimum automnal. Les trois secteurs susmentionnés font partie du rhithron. Il est nécessaire, toutefois, d'étendre le sens donné par Illies et Botosaneanu (1961) au mot rhithron pour l'appliquer au cours d'eau libanais.

Sept stations furent prospectées le long du cours d'eau :

Station 1 - Secteur I. Altitude $1250 \mathrm{~m}$. Le cours d'eau est actif depuis novembre décembre jusqu'à juin-août, selon les années. Plusieurs points de prélèvements furent prospectés, dont certains irrégulièrement.

Station 2 - A la limite du secteur I. Altitude $1050 \mathrm{~m}$. Le ruisseau est temporaire, il est actif depuis décembre jusqu'à juin-juillet, selon les années.

Station 3. A la limite du secteur II. Altitude $1020 \mathrm{~m}$. Apparition de petites résurgences; le ruisseau est pérenne.

Station 4 - Secteur II. Altitude $1000 \mathrm{~m}$. Zone des sources pérennes.

Station 5 - Secteur II. A l'amont d'un barrage hydroélectrique.
Station 6 - Secteur II. La rivière, après avoir collecté toutes les sources et résurgences.

Station 7 - A la limite du secteur II, côté aval. Altitude $920 \mathrm{~m}$, au niveau de la ville de Oab Ilias, après avoir reçu des ordures ménagères et certains produits polluants.

Une huitième station, à la limite du secteur III, a été prospectée sporadiquement. Les relevés sont insuffisants et ne font pas partie de la présente étude.

\section{4. - Technique et matériels}

Les deux stations 4 et 7 ont été prospectées avec un filet de Surber de $0,1 \mathrm{~m}^{2}$ de surface et de $0,3 \mathrm{~mm}$ environ de vide de maille, à raison d'un prélèvement mensuel entre juin 1973 et octobre 1975 inclus et de trois prélèvements mensuels par station entre juillet 1977 et janvier 1979 inclus.

Les stations 2, 3, 5 et 6 furent prospectées spora. diquement entre 1973 et 1975 et méthodiquement en 1977-1979, à raison de trois prélèvernents, par station, avec le filet de Surber. La station 1 fut prospectée durant les deux saisons humides 78-79 et 79.80 avec un filet quelconque à bord droit, de $0.3 \mathrm{~mm}$ de vide maille, jusqu'à obtenir un échantillon suffisamment riche en représentants de la faune; cette station est formée de deux points de prélèvement.

L'échantillon était mis directement dans l'alcool, le tri se faisait quelques jours à une semaine plus tard au laboratoire. On s'est borné à reconnaître le groupe zoologique dans certains cas, le genre pour les Amphipades, les Ephéméroptères et les Plécoptères et l'espèce chaque fois que possible.

\section{5. - Résultats}

Les 180 échantillons, récoltés entre 1973 et 1980 dans les sept premières stations, ont donné 155027 individus répartis de la façon suivante : 98490 larves d'Ephéméroptères, 3404 larves de Plécoptères, 4157 larves de Trichoptères, 12867 larves de Diptères, 1556 larves et adultes Coléoptères et $40 \mathrm{lar}-$ ves d'Odonates : 25873 Gammares, 1821 Ostracodes et 182 Hydracariens, 3640 Ancyles, 1871 Oli- 
gochètes, 387 Hirudinés, 211 Planaires et 528 individus appartenant à d'autres groupes. Le total des récoltes des années 1977.1980 , par station, est con. signé dans le tableau II. Les relevés mensuels des Plécoptères et des Ephéméroptères, sur une année et dans cinq stations représentatives, sont consignés dans le tableau III. Les paramètres physicochimiques d'un des points de la station 1 sont indiqués dans le tableau I (les relevés concernant les autres stations ont été perdus durant l'occupation et le pillage de la Faculté des Sciences en été 1982).

Le matériel récolté (macroinvertébrés) met en relief la très nette tendance des Plécoptères à diminuer au fur et à mesure que l'on s'éloigne du secteur montagnard vers la zone inframontagnarde, excepté au niveau de la station 6. Les Ephéméroptères, quant à eux, augmentent en nombre depuis la station 1 jusqu'à la station 5 , puis commencent à diminuer (Tableau V).

Tableau 1. Relevés physico-chimiques se rapportant à la première station.

A.T $=$ alcalinité totale $;$ D. $\mathrm{T}$. $=$ dureté totale $;$ D.P. $=$ dureté partielle.

Les résultats, en ppm, sont exprimés en équivalent $\mathrm{CaCO}_{3}$. La silice est exprimée en ppm.

\begin{tabular}{lcccccc}
\hline Date & $\mathrm{t}^{\circ}$ & pH & A.T. & D.T. & D.P. & Silice \\
\hline $14 / 11 / 79$ & & 7,8 & 140 & 160 & 145 & 6 \\
$10 / 12 / 79$ & 12 & 7,6 & & 140 & 130 & \\
$17 / 1 / 80$ & 10,4 & 7,9 & 120 & 120 & 110 & \\
$13 / 2 / 80$ & 11,2 & 8,2 & 160 & 150 & 135 & \\
$11 / 3 / 80$ & 9,8 & 8,1 & 100 & 105 & 90 & 6,5 \\
$2 / 4 / 80$ & 10,2 & 7,8 & 135 & 140 & 120 & 6 \\
$22 / 4 / 80$ & 13,5 & 7,7 & 120 & 135 & 125 & 6 \\
$22 / 5 / 80$ & 14 & 7,8 & 150 & 165 & 155 & 5,2 \\
$12 / 6 / 80$ & 14,3 & 8,1 & 160 & 175 & 150 & 7 \\
\hline
\end{tabular}

Tableau II. Matériel récolté dans les 7 stations entre 1977 et 1980 .

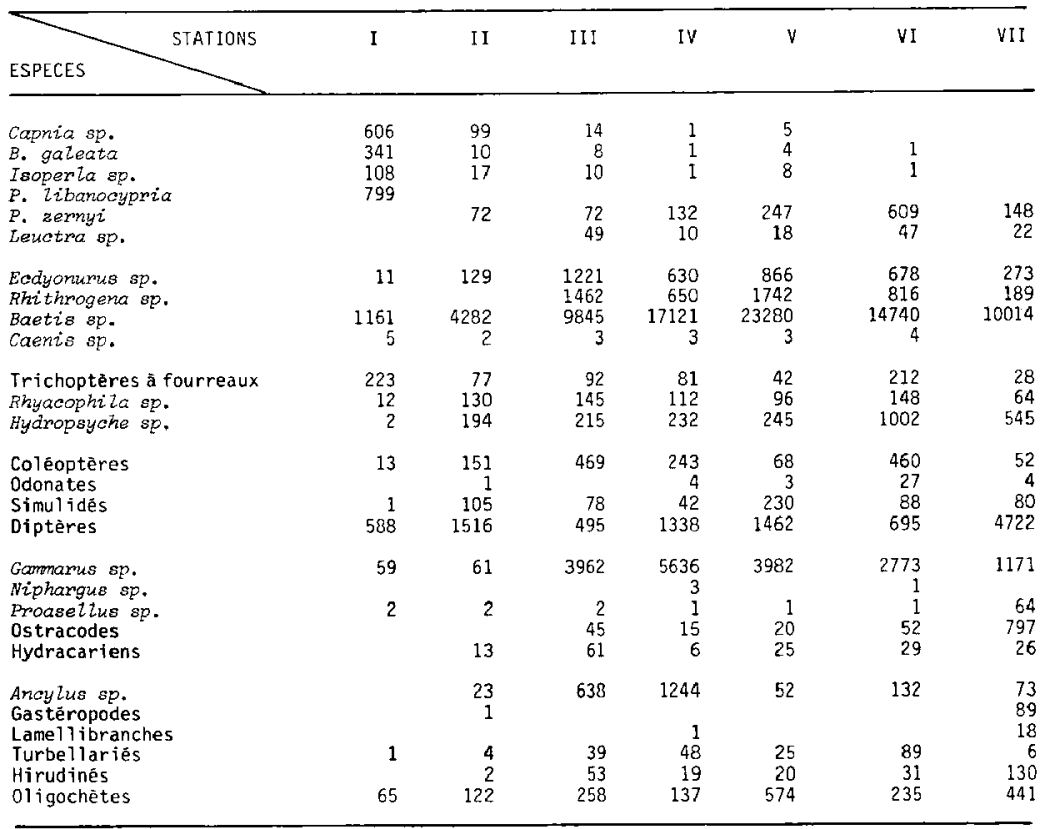


Tableau III. Matériel récolté à différents niveaux du cours d'eau de Qab Ilias.

\begin{tabular}{|c|c|c|c|c|c|c|c|c|c|c|}
\hline $\begin{array}{l}\text { 省 } \\
\text { 品 }\end{array}$ & 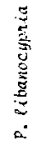 & $\begin{array}{l}\frac{\dot{0}}{8} \\
\frac{3}{3} \\
\frac{2}{2}\end{array}$ & 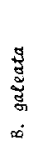 & 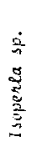 & 㺃 & 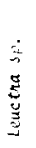 & $\begin{array}{l}\vdots \\
0 \\
3 \\
\vdots \\
0 \\
0\end{array}$ & 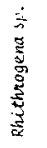 & 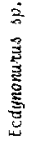 & \begin{tabular}{l}
$\frac{j}{3}$ \\
3 \\
$\vdots$ \\
\multirow{3}{*}{}
\end{tabular} \\
\hline \multicolumn{11}{|c|}{ STATION 1} \\
\hline $\begin{array}{r}11 / 79 \\
12 / 79 \\
1 / 80 \\
2 / 80 \\
3 / 80 \\
4 / 80 \\
5 / 80\end{array}$ & $\begin{array}{r}59 \\
164 \\
61 \\
45\end{array}$ & $\begin{array}{r}249 \\
68 \\
89 \\
20\end{array}$ & $\begin{array}{r}47 \\
36 \\
12 \\
2\end{array}$ & $\begin{array}{r}1 \\
1 \\
22 \\
24\end{array}$ & & & $I$ & & $\begin{array}{l}1 \\
4 \\
1\end{array}$ & $\begin{array}{r}13 \\
2 \\
7 \\
7 \\
2 \\
4 \\
751\end{array}$ \\
\hline \multicolumn{11}{|c|}{ STATION 2} \\
\hline $\begin{array}{r}12 / 77 \\
1 / 78 \\
2 / 78 \\
3 / 78 \\
4 / 78 \\
5 / 78\end{array}$ & & $\begin{array}{l}11 \\
33 \\
12\end{array}$ & $\begin{array}{l}9 \\
1\end{array}$ & $\begin{array}{l}1 \\
1 \\
9 \\
6\end{array}$ & $\begin{array}{r}6 \\
5 \\
10\end{array}$ & & 1 & & $\begin{array}{r}1 \\
2 \\
10\end{array}$ & $\begin{array}{r}4 \\
4 \\
5 \\
1 \\
309 \\
3287\end{array}$ \\
\hline \multicolumn{11}{|c|}{ STATION 3} \\
\hline $\begin{array}{r}1 / 78 \\
2 / 78 \\
3 / 78 \\
4 / 78 \\
5 / 78 \\
6 / 78 \\
7 / 78 \\
8 / 78 \\
9 / 78 \\
10 / 78 \\
11 / 78 \\
12 / 78\end{array}$ & & 4 & 1 & $\begin{array}{l}2 \\
5 \\
3\end{array}$ & $\begin{array}{l}7 \\
2 \\
1\end{array}$ & $\begin{array}{l}1 \\
3 \\
5 \\
4 \\
5\end{array}$ & $\begin{array}{l}1 \\
2\end{array}$ & $\begin{array}{r}2 \\
25 \\
185 \\
21 \\
94 \\
250 \\
213\end{array}$ & $\begin{array}{r}10 \\
6 \\
1 \\
11 \\
12 \\
3 \\
19 \\
8 \\
339 \\
213 \\
157 \\
63\end{array}$ & $\begin{array}{r}12 \\
3 \\
3 \\
369 \\
2341 \\
924 \\
397 \\
998 \\
347 \\
217 \\
134 \\
32\end{array}$ \\
\hline \multicolumn{11}{|c|}{ STATION 5} \\
\hline $\begin{array}{r}1 / 78 \\
2 / 78 \\
3 / 78 \\
4 / 78 \\
5 / 78 \\
6 / 78 \\
7 / 78 \\
8 / 78 \\
9 / 78 \\
10 / 78 \\
11 / 78 \\
12 / 78\end{array}$ & & 3 & 3 & $\begin{array}{l}3 \\
3 \\
2\end{array}$ & $\begin{array}{r}1 \\
1 \\
5 \\
3 \\
\\
17 \\
8 \\
8 \\
9 \\
21\end{array}$ & 4 & 2 & $\begin{array}{r}36 \\
2 \\
1 \\
1 \\
\\
2 \\
30 \\
38 \\
152 \\
83 \\
366 \\
398\end{array}$ & $\begin{array}{r}24 \\
5 \\
1 \\
15 \\
15 \\
16 \\
11 \\
5 \\
26 \\
12 \\
63 \\
8 \\
88\end{array}$ & $\begin{array}{r}48 \\
9 \\
12 \\
557 \\
4115 \\
3134 \\
3618 \\
2177 \\
1069 \\
1136 \\
1036 \\
462\end{array}$ \\
\hline \multicolumn{11}{|c|}{ STATION 6} \\
\hline $\begin{array}{r}1 / 78 \\
2 / 78 \\
3 / 78 \\
4 / 78 \\
5 / 78 \\
6 / 78 \\
7 / 78 \\
8 / 78 \\
9 / 78 \\
10 / 78 \\
11 / 78 \\
12 / 78\end{array}$ & & & 1 & 1 & $\begin{array}{r}41 \\
8 \\
2 \\
1 \\
1 \\
21 \\
8 \\
15 \\
106 \\
5 \\
24 \\
20\end{array}$ & $\begin{array}{l}7 \\
1\end{array}$ & $\begin{array}{l}1 \\
2\end{array}$ & $\begin{array}{r}62 \\
26 \\
\\
3 \\
1 \\
1 \\
2 \\
17 \\
15 \\
105 \\
128 \\
100\end{array}$ & $\begin{array}{r}102 \\
23 \\
20 \\
12 \\
14 \\
14 \\
29 \\
22 \\
36 \\
20 \\
42 \\
24\end{array}$ & $\begin{array}{r}143 \\
52 \\
42 \\
446 \\
1376 \\
1650 \\
1222 \\
1662 \\
1121 \\
705 \\
490 \\
241\end{array}$ \\
\hline
\end{tabular}


Une analyse des différents taxa montre la présence de quatre groupes écologiques:

- Groupe I: Protonemura libanocypria Zwick, Capnia sp., Brachyptera galeata Koponen et Isoperla sp.. Il est dominant dans le secteur montagnard

- Groupe II : Protonemura zernyi Aubert et Leuctra sp. Sa proportion est faible, elle at teint le maximum de $4 \%$ au niveau de la station 6.

- Groupe III : Rhithrogena sp. et Ecdyonurus sp.. Il est caractéristique du secteur submontagnard.

- Groupe IV : Baetis sp. et Caenis sp.. Il est dominant dans toutes les stations exceptée la première.

Leur évolution (tableau VI) met en relief la prédominence du groupe I dans le secteur montagnard et la tendance du groupe II à augmenter de l'amont vers l'aval de la zone submontagnarde (Tableau VII). Le groupe III paraît trouver son optimum dans le secteur submontagnard. Enfin, le groupe IV paraît fluctuer en proportion inverse aux autres groupes, ses effectifs vont toujours en augmentant, la baisse at niveau de la station 7 peut être imputée à l'influence humaine.

Tableau IV. Nombre et proportion de Plécoptères et d'Ephéméroptères récoltés par Ulfstrand en Scandinavie, Elliott en Angleterre et Lavandier et Dumas en France.

\begin{tabular}{lrrrr}
\hline & \multicolumn{2}{c}{ Plécoptères } & \multicolumn{2}{c}{ Ephéméroptères } \\
& $\mathrm{Nb}$ & $\%$ & $\mathrm{Nb}$ & $\%$ \\
\hline Scandinavie & 5925 & $68 \%$ & 2789 & $32 \%$ \\
Angleterre & 10848 & $56 \%$ & 8597 & $44 \%$ \\
France & 20407 & $68 \%$ & 9735 & $32 \%$ \\
\multicolumn{2}{c}{ (15 503 individus appartiennent à une } \\
seule espèce)
\end{tabular}

Tableau V. Nombre et proportion de Plécoptères et d'Ephéméroptères récoltés dans les 7 stations de la rivière Qab Ilias.

\begin{tabular}{lccccc}
\hline \multirow{2}{*}{ Station } & Nb & \multicolumn{4}{c}{ Plécoptères } \\
d'échantillons & $\mathrm{Nb}$ & $\%$ & $\mathrm{Nb}$ & $\%$ \\
\hline I & 13 & 1854 & 61 & 1177 & 39 \\
II & 10 & 155 & 3,5 & 4413 & 96,5 \\
III & 20 & 153 & 1,5 & 12531 & 98,5 \\
IV & 20 & 145 & 0,8 & 18404 & 99,2 \\
V & 20 & 282 & 1 & 25911 & 99 \\
VI & 20 & 658 & 4 & 16200 & 96 \\
VII & 20 & 170 & 1 & 10376 & 99 \\
\hline
\end{tabular}

Tableau VI. Evolution de la proportion des quatre groupes dans les 7 stations

\begin{tabular}{lcccc}
\hline Station & $\begin{array}{c}\text { groupe I } \\
\%\end{array}$ & $\begin{array}{c}\text { groupe II } \\
\%\end{array}$ & $\begin{array}{c}\text { groupe III } \\
\%\end{array}$ & $\begin{array}{c}\text { groupe IV } \\
\%\end{array}$ \\
\hline I & 61 & 0 & 0,5 & 38,5 \\
II & 1,5 & 2 & 3 & 93,5 \\
III & 0,5 & 1 & 21 & 77,5 \\
IV & 0 & 0,7 & 9,3 & 90 \\
V & 0 & 1 & 10 & 89 \\
VI & 0 & 4 & 9 & 87 \\
VII & 0 & 1 & 5 & 94 \\
\hline
\end{tabular}

Tableau VIII. Pourcentages des Plécoptères d'amont (groupe I) et d'aval (groupe II) dans les sept stations.

\begin{tabular}{lrrrrrrr}
\hline Station & I & II & III & \multicolumn{1}{c}{ IV } & \multicolumn{1}{c}{ V } & VI & VII \\
\hline Groupe I & 100 & 54 & 21 & 1.4 & 6 & 0.3 & 0 \\
Groupe II & 0 & 46 & 79 & 98,6 & 94 & 99,7 & 100 \\
\hline
\end{tabular}

\section{6. - Conclusion}

La presque totalité de la faune du secteur montagnard est formée d'insectes qui surmontent, d'une façon ou d'une autre, la sécheresse estivale. Les Plécoptères dominent à ce niveau. Le rythme annuel du débit ne permet pas aux autres insectes de survivre. La zone montagnarde est le refuge des Plécoptères où aucun autre macroinvertébrés ne peut rivaliser, excepté, peut-être, les Diptères à croissance rapide. Le secteur submontagnard est caractérisé par la relative stabilité du débit l'année durant. Sa colonisation par d'autres groupes d'insectes est ainsi possible. Les Plécoptères, ne pouvant pas soutenir la concurrence, sont éliminés. Nous estimons que la sécheresse estivale et le rythme qu'elle engendre sont les facteurs déterminants permettant de definir les deux secteurs susmentionnés. Les caractéristiques de la zone inframontagnarde restent à être définies.

La division du bassin hydrographique de Qab Ilias en différents secteurs, en fonction de l'altitude, est comparable, d'une certaine façon, à la zonation biologique des cours d'eau observée en Europe. L'altitude et la latitude joueraient un rôle comparable. La division du rhithron, dans le sens de biocénose de cours d'eau de montagne à grande vitesse d'écoulement, en trois zones n'est valable, pour le moment, que pour le Nahr Qab Ilias. Des prospections métho- 
diques dans d'autres bassins hydrographiques du Liban et la connaissance du cycle de vie des composants de la zoocénose permettront une meilleure définition de ces termes.

\section{Travaux cités}

Elliott (J.M.). 1967. - The life-history and drifting of the Plecoptera and Epherneroptera in a Dartmoor stream. J. Anim. Ecol., $36: 343.362$.
Illies (3.) et Botosaneanu (L.). 1961. - Problemes et méthodes de la classification et de la zonation ecologique des eaux courantes, considérées surtout du point de vue faunistique. Mitt. Intermal. Verein. Limmol., $12: 1-57$.

Lavandier (P.) et Dumas (J.). 1971 . - Microrépartition des invertébrés benthiques dans des ruisseaux des Pyrénées centrales. Annals Limnol, 7 (1): 7-23.

Legier (P.) et Talin (J.). 1973. - Comparaison de ruisseaux permanents et temporaires de la Provence calcaire. Annls Limnol. 9 (3) : 273-292.

Ulfstrand (S.). 1968. - Life cycles of benthic insects in Lapland streams (Ephemeroptera. Plecoptera, Trichoptera, Diptera Simuliidea). Oikos, 19 (2): $167-190$.

Verneaux (J.) et Tuffery (G.). 1967. - Une méthode zoologique pratique de dètermination de la qualité biologique des eaux courantes. Ann. Sc. Univ. Besançon, C3:79.90. 